\title{
Non-contiguous finished genome sequence and description of Anaerococcus vaginalis
}

\author{
Perrine Hugon ${ }^{1}$, Ajay Kumar Mishra ${ }^{1}$, Catherine Robert ${ }^{1}$, Didier Raoult ${ }^{1}$ and Pierre-Edouard \\ Fournier $^{1 *}$ \\ ${ }^{1}$ Unité de Recherche sur les Maladies Infectieuses et Tropicales Emergentes, UMR CNRS \\ 6236 - IRD 198, Faculté de médecine, Aix-Marseille Université \\ *Corresponding author: Pierre-Edouard Fournier (pierre-edouard.fournier@univmed.fr) \\ Keywords: Anaerococcus vaginalis, genome
}

\begin{abstract}
We report the properties of a draft genome sequence of the bacterium Anaerococcus vaginalis strain PH9, a species within the Anaerococcus genus. This strain, whose genome is described here, was isolated from the fecal flora of a 26-year-old woman suffering from morbid obesity. A. vaginalis is an obligate anaerobic coccus. Here we describe the features of this organism, together with the complete genome sequence and annotation. The 2,048,125-bp long (one chromosome but no plasmid) and contains 2,095 protein-coding and 38 RNA genes, including three rRNA genes.
\end{abstract}

\section{Introduction}

Anaerococcus vaginalis strain PH9 (= CSUR P188= DSM25446) was isolated from the stool of a 26-yearold woman suffering from morbid obesity as part of a study aiming at cultivating all species within human feces. It is a Gram-positive, anaerobic, indolenegative coccus.

The genus Anaerococcus (Ezaki et al. 2001) was created in 2001 [1] and to date, this genus consist of saccharolytic, butyrate-producing anaerobic and non-motile gram-positive cocci. Seven species are validated, including $A$. hydrogenalis, $A$. lactolyticus, $A$. murdochii, A. octavius, A. prevotii, $A$. tetradius and $A$. vaginalis $[2,3]$. Members of the genus Anaerococcus have mostly been isolated from the human vagina, but have also occasionally been identified in the nasal cavity, on the skin, and in various infectious processes including ovarian, peritoneal, sacral, digital and cervical abscesses, vaginoses, bacteremias, foot ulcers, a sternal wound, and a knee arthritis [1-5]. In addition, uncultured bacteria with $16 \mathrm{~S}$ rRNA sequences highly similar to members of the Anaerococcus genus have been detected in metagenomes from the human skin flora [6].

A. vaginalis (Li et al. 1992) was first isolated from vaginal discharges and ovarian abscesses [7]. Initially, it was classified in the genus Peptostreptococcus but later reclassified within the genus Anaerococcus $[1]$.

To the best of our knowledge, we first report the isolation of Anaerococcus sp. from the fecal flora of a patient suffering from morbid obesity. Herein, we present a set of features for of $A$. vaginalis strain PH9 together with the description of the complete genomic sequence and annotation.

\section{Classification and features}

A stool sample was collected from a 26-year-old woman living in Marseille, France, who suffered from morbid obesity: BMI=48.2 (118.8 kg, 1.57 meter). At the time of stool sample collection, she was not a drug-user and was not on a diet. The patient gave an informed and signed consent, and the agreement of local ethics committee of the IFR48 (Marseille, France) were obtained under agreement 11-017. The fecal specimen was preserved at $-80^{\circ} \mathrm{C}$ after collection. Strain PH9 (Table 1) was isolated in 2011 by anaerobic cultivation on 5\% sheep bloodenriched Columbia agar (BioMerieux, Marcy l'Etoile, France) after 4 days of preincubation of the stool sample with addition of thioglycolate in a blood culture bottle.

This strain exhibited a 98.8\% $16 \mathrm{~S}$ rRNA sequence similarity with $A$. vaginalis (Li et al. 1992), the phylogenetically closest validated Anaerococcus species (Figure 1). This value was higher than the 98.7\% 16S rRNA gene sequence threshold recommended by Stackebrandt and Ebers to delineate a new species [18]. As a consequence, strain PH9 belongs to $A$. vaginalis. 
Growth at different temperatures $\left(25,30,37,45^{\circ} \mathrm{C}\right)$ was tested; no growth occurred at $25^{\circ} \mathrm{C}$ and $45^{\circ} \mathrm{C}$, growth occurred between 30 and $37^{\circ} \mathrm{C}$, and optimal growth was observed at $37^{\circ} \mathrm{C}$. Colonies were 0.5 $\mathrm{mm}$ to $1 \mathrm{~mm}$ in diameter on blood-enriched Columbia agar and Brain Heart Infusion (BHI) agar. Growth of the strain was tested under anaerobic and microaerophilic conditions using GENbag anaer and GENbag microaer systems, respectively (BioMérieux), and in the presence of air, with or without $5 \% \mathrm{CO}_{2}$ and in aerobic conditions. Optimal growth was obtained anaerobically, with weak growth being observed in microaerophilic condition, and no growth occurring in aerobic conditions and with $5 \% \mathrm{CO}_{2}$. Gram staining showed Gram positive cocci. A motility test was negative. Cells grown on agar are Gram-positive (Figure 2) and have a mean diameter of $0.71 \mu \mathrm{m}$ by electron microscopy and are mostly grouped in pairs, short chains or small clumps (Figure 3).

Table 1. Classification and general features of Anaerococcus vaginalis strain $\mathrm{PH} 9$

\begin{tabular}{|c|c|c|c|}
\hline MIGS ID & Property & Term & Evidence code $^{\mathrm{a}}$ \\
\hline & \multirow{8}{*}{ Current classification } & Domain Bacteria & TAS [8] \\
\hline & & Phylum Firmicutes & TAS [9-11] \\
\hline & & Class Clostridia & TAS $[12,13]$ \\
\hline & & Order Clostridiales & TAS $[14,15]$ \\
\hline & & Family Clostridiales family XI Incertae sedis & TAS [16] \\
\hline & & Genus Anaerococcus & TAS [1] \\
\hline & & Species Anaerococcus vaginalis & IDA \\
\hline & & Type strain PH9 & \\
\hline & Gram stain & positive & IDA \\
\hline & Cell shape & coccoid & IDA \\
\hline & Motility & nonmotile & IDA \\
\hline & Sporulation & nonsporulating & IDA \\
\hline & Temperature range & mesophile & IDA \\
\hline & Optimum temperature & $37^{\circ} \mathrm{C}$ & IDA \\
\hline MIGS-6.3 & Salinity & growth in $\mathrm{BHI}$ medium $+5 \% \mathrm{NaCl}$ & IDA \\
\hline \multirow[t]{3}{*}{ MIGS-22 } & Oxygen requirement & anaerobic & IDA \\
\hline & Carbon source & unknown & \\
\hline & Energy source & peptones & NAS \\
\hline MIGS-6 & Habitat & human gut & IDA \\
\hline \multirow[t]{3}{*}{ MIGS-15 } & Biotic relationship & free living & IDA \\
\hline & Pathogenicity & Unknown & \\
\hline & Biosafety level & 2 & \\
\hline MIGS-14 & Isolation & human feces & \\
\hline MIGS-4 & Geographic location & France & IDA \\
\hline MIGS-5 & Sample collection time & January 2011 & IDA \\
\hline MIGS-4.1 & Latitude & 43.296482 & IDA \\
\hline MIGS-4.1 & Longitude & 5.36978 & IDA \\
\hline MIGS-4.3 & Depth & surface & IDA \\
\hline MIGS-4.4 & Altitude & $0 \mathrm{~m}$ above sea level & IDA \\
\hline
\end{tabular}

Evidence codes - IDA: Inferred from Direct Assay; TAS: Traceable Author Statement (i.e., a direct report exists in the literature); NAS: Non-traceable Author Statement (i.e., not directly observed for the living, isolated sample, but based on a generally accepted property for the species, or anecdotal evidence). These evidence codes are from the Gene Ontology project [17]. If the evidence is IDA, then the property was directly observed for a live isolate by one of the authors or an expert mentioned in the acknowledgements. 


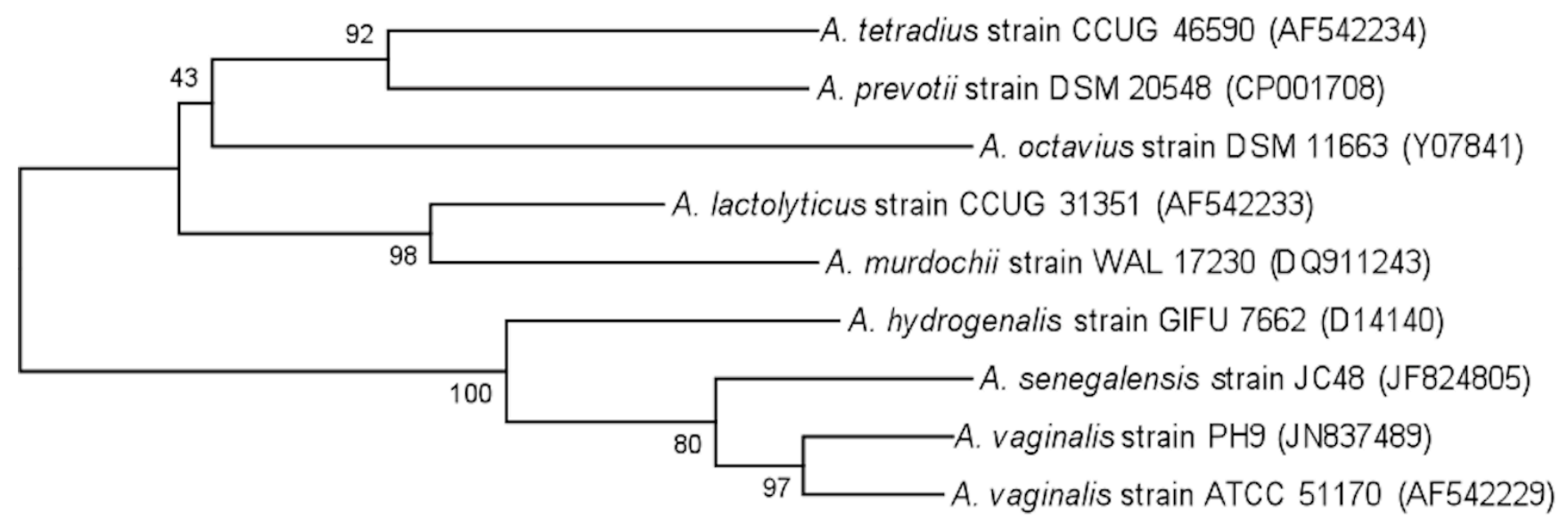

Figure 1. Phylogenetic tree highlighting the position of Anaerococcus vaginalis strain $\mathrm{PH} 9$ relative to other type strains within the Anaerococcus genus. GenBank accession numbers are indicated in parentheses. Sequences were aligned using CLUSTALW, and phylogenetic inferences obtained using the maximum-likelihood method and the MEGA software. Numbers at the nodes are bootstrap values obtained by repeating 500 times the analysis to generate a majority consensus tree. The scale bar represents a $1 \%$ nucleotide sequence divergence.

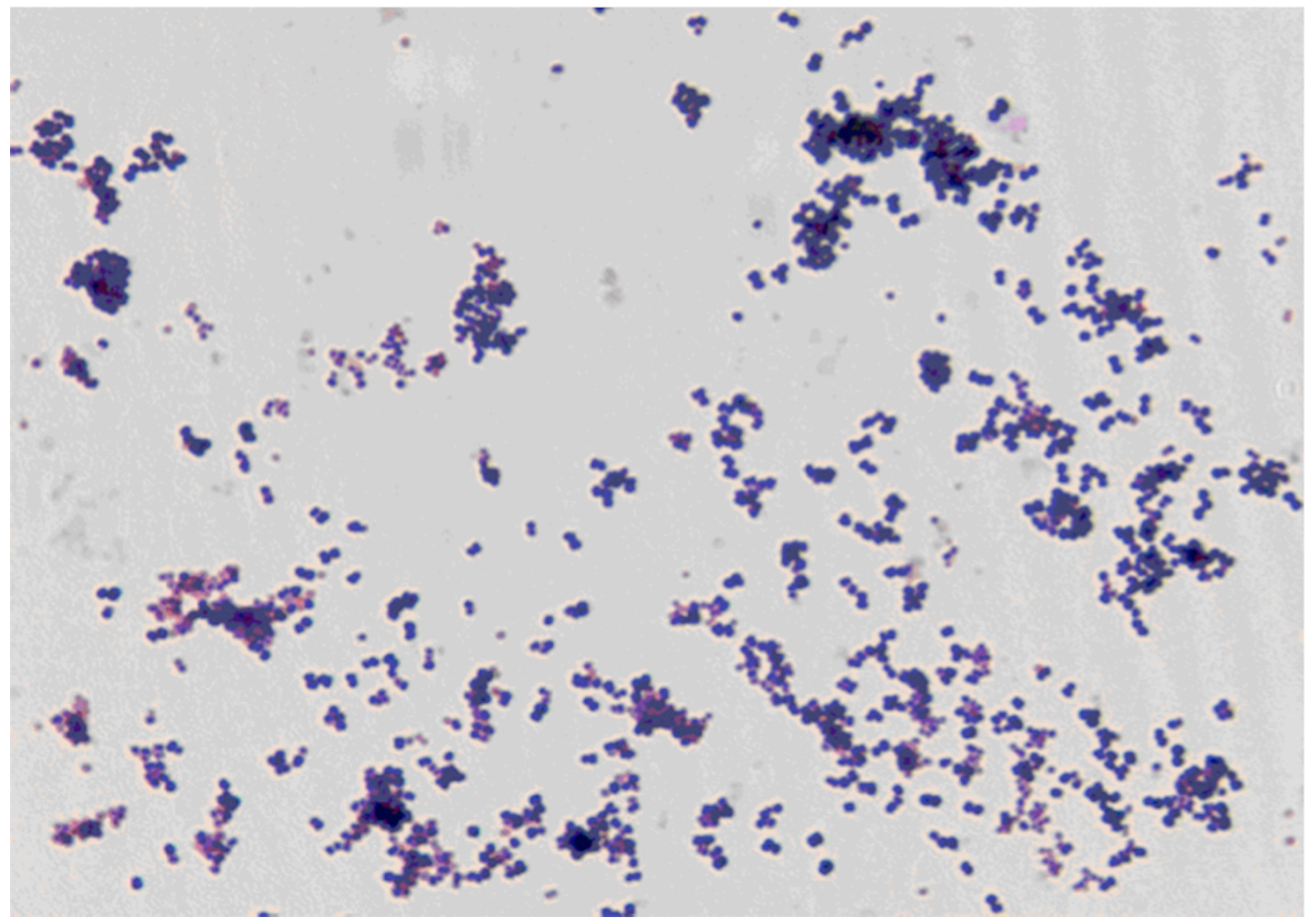

Figure 2. Gram staining of $A$. vaginalis strain $\mathrm{PH} 9$ 


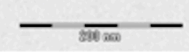

Figure 3. Transmission electron microscopy of $A$. vaginalis strain $\mathrm{PH} 9$, using a Morgani 268D (Philips) at an operating voltage of $60 \mathrm{kV}$. The scale bar represents $900 \mathrm{~nm}$.

Strain PH9 exhibited catalase activity but no oxidase activity. Using API Rapid ID 32A, a positive reaction was observed for arginine dihydrolase, histidine arylamidase, leucine arylamidase and mannose fermentation. A weak activity was observed for glycine arylamidase. A. vaginalis is susceptible to penicillin $\mathrm{G}$, imipeneme, amoxicillin + clavulanic acid, vancomycin, clindamycin and metronidazole.

Matrix-assisted laser-desorption/ionization timeof-flight (MALDI-TOF) MS protein analysis was carried out as previously described [19]. Briefly, a pipette tip was used to pick one isolated bacterial colony from a culture agar plate, and to spread it as a thin film on a MTP 384 MALDI-TOF target plate (Bruker Daltonics, Germany). Twelve distinct deposits were done for strain PH9 from twelve isolated colonies. Each smear was overlaid with $2 \mu \mathrm{L}$ of matrix solution (saturated solution of alpha-cyano4-hydroxycinnamic acid) in 50\% acetonitrile, 2.5\% tri-fluoracetic acid, and allowed to dry for five minutes. Measurements were performed with a Microflex spectrometer (Bruker). Spectra were recorded in the positive linear mode for the mass range of 2,000 to 20,000 $\mathrm{Da}$ (parameter settings: ion source 1 (ISI), 20kV; IS2, $18.5 \mathrm{kV}$; lens, $7 \mathrm{kV}$ ). A spectrum was obtained after 675 shots at a variable laser power. The time of acquisition was between 30 seconds and 1 minute per spot. The twelve PH9 spectra were imported into the MALDI Bio Typer software (version 2.0, Bruker) and analyzed by standard pattern matching (with default parameter settings) against the main spectra of 2,843 bacteria, including spectra from seven validated Anaerococcus species used as reference data, in the Bio Typer database. The method of identification includes the $\mathrm{m} / \mathrm{z}$ from 3,000 to $15,000 \mathrm{Da}$. For every spectrum, 100 peaks at most were taken into account and compared with the spectra in the database. A score enabled the presumptive identification and discrimination of the tested species from those in the database: a score $\geq 2$ with a validated species enabled the identification at the species level; a score $\geq 1.7$ but $<2$ enabled the identification at the genus level; and a score $<1.7$ did not enable any identification. Spectra were compared with the Bruker database that contained spectra from the seven validated Anaerococcus species. The score and spectra obtained were similar to those of $A$. vaginalis, thus confirming that our isolate was a member of the $A$. vaginalis species. We incremented our database with the spectrum from strain PH9 (Figure 4). 


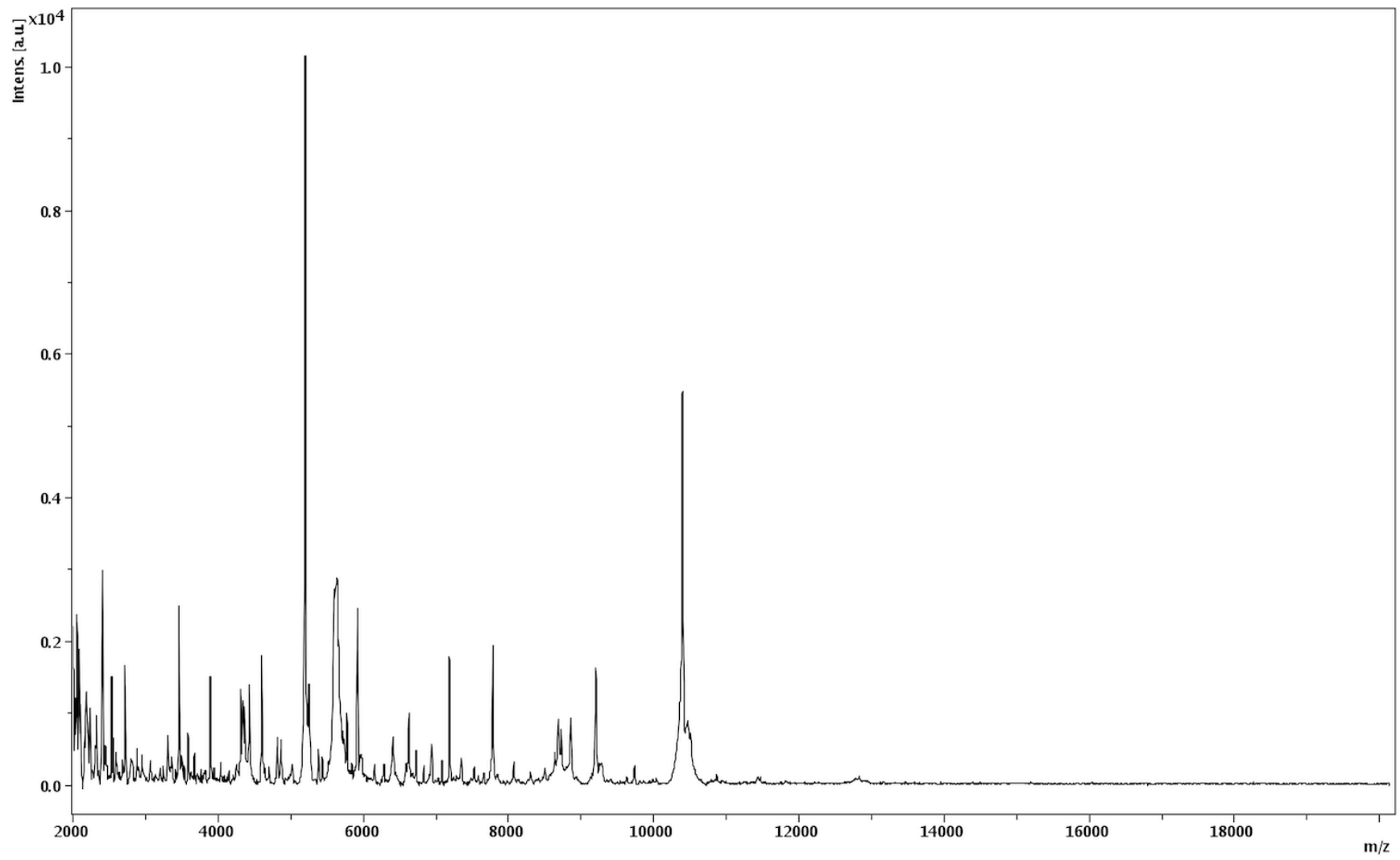

Figure 4: Reference mass spectrum from A. vaginalis strain PH9. Spectra from 12 individual colonies were compared and a reference spectrum was generated.

Table 2. Project information

\begin{tabular}{lll}
\hline MIGS ID & Property & Term \\
\hline MIGS-31 & Finishing quality & High-quality draft \\
MIGS-28 & Libraries used & One 454 paired end 3-kb library \\
MIGS-29 & Sequencing platforms & 454 GS FLX Titanium \\
MIGS-31.2 & Fold coverage & 35 \\
MIGS-30 & Assemblers & Newbler version 2.5.3 \\
MIGS-32 & Gene calling method & Prodigal \\
& INSDC ID & 2000019206 \\
& NCBI project ID & CAGU00000000 \\
& Genbank Date of Release & 31-05-2012 \\
& Gold ID & Gi13719 \\
MIGS-13 & Project relevance & Study of the human gut microbiome \\
\hline
\end{tabular}




\section{Genome sequencing and annotation Genome project history}

The organism was selected for sequencing on the basis of its phylogenetic position and 16S rRNA similarity to other members of the Anaerococcus genus, and is part of a study of the human digestive flora aiming at isolating all bacterial species within human feces. It is the third published genome from an Anaerococcus species and the first genome from the $A$. vaginalis species. A summary of the project information is shown in Table 2 . The Genbank accession number is CAGU00000000. The genome consists of 93 contigs. Table 2 shows the project information and its association with MIGS version 2.0 compliance [1].

\section{Growth conditions and DNA isolation}

A. vaginalis strain PH9 (DSM25446, CSUR P188) was grown anaerobically on $5 \%$ sheep bloodenriched Columbia agar at $37^{\circ} \mathrm{C}$. Six petri dishes were spread and resuspended in $6 \times 100 \mu$ of G2 buffer (EZ1 DNA Tissue kit, Qiagen). A first mechanical lysis was performed by glass powder on the Fastprep-24 device (Sample Preparation system from MP Biomedicals, USA) for 40 seconds. DNA was then incubated for a lysozyme treatment (30 minutes at $37^{\circ} \mathrm{C}$ ) and extracted using the BioRobot EZ 1 Advanced XL (Qiagen).The DNA was then concentrated and purified using the Qiamp kit (Qiagen). The yield and the concentration was measured by the Quant-it Picogreen kit (Invitrogen) on the Genios_Tecan fluorometer at $115.2 \mathrm{ng} / \mu \mathrm{l}$.

\section{Genome sequencing and assembly}

Five $\mu \mathrm{g}$ of DNA were mechanically fragmented on the Hydroshear device (Digilab, Holliston, MA,USA) with an enrichment size at 3-4kb. The DNA fragmentation was visualized through the Agilent 2100 BioAnalyzer on a DNA labchip 7500 with an optimal size of $2.92 \mathrm{~kb}$. The library was constructed according to the 454 GS FLX Titanium paired end protocol. Circularization and nebulization were performed and generated a pattern with an optimal at $415 \mathrm{bp}$. After PCR amplification through $15 \mathrm{cy}-$ cles followed by double size selection, the single stranded paired-end library was then quantified on the Quant-it Ribogreen kit (Invitrogen) on the Genios Tecan fluorometer at $1,440 \mathrm{pg} / \mu \mathrm{L}$. The library concentration equivalence was calculated as
$6.36 \mathrm{E}+09$ molecules/ $\mu \mathrm{L}$. The library was stored at $20^{\circ} \mathrm{C}$ until further use.

The library was clonally amplified with $0.25 \mathrm{cpb}$ and $1 \mathrm{cpb}$ respectively in $2 \times 8$ emPCR reactions with the GS Titanium SV emPCR Kit (Lib-L) v2 (Roche). The yields of the emPCR were quite high at $17.78 \%$ but in the range of 5 to $20 \%$ from the Roche procedure.

Approximately 790,000 beads were loaded on the GS Titanium PicoTiterPlate PTP Kit 70x75 and sequenced with the GS FLX Titanium Sequencing Kit XLR70 (Roche). The run was performed overnight and then analyzed on the cluster through the gsRunBrowser and Newbler assembler (Roche). A total of 191,750 passed filter wells were obtained and generated 59.42 $\mathrm{Mb}$ with an average length of $309 \mathrm{bp}$. The passed filter sequences were assembled Using Newbler with $90 \%$ identity and 40bp as overlap. The final assembly identified 93 large contigs (>1500bp).

\section{Genome annotation}

Open Reading Frames (ORFs) were predicted using Prodigal [20] with default parameters but the predicted ORFs were excluded if they were spanning a sequencing GAP region. The predicted bacterial protein sequences were searched against the GenBank database [21] and the Clusters of Orthologous Groups (COG) databases using BLASTP. The tRNAScanSE tool [22] was used to find tRNA genes, whereas ribosomal RNAs were found by using RNAmmer [23] and BLASTn against the NR database. ORFans were identified if their BLASTP $E$-value were lower than 1e-03 for alignment length greater than 80 amino acids. If alignment lengths were smaller than 80 amino acids, we used an $E$-value of 1e-05. Such parameter thresholds have already been used in previous works to define ORFans. To estimate the mean level of nucleotide sequence similarity at the genome level between Anaerococcus species, we compared the ORFs only using BLASTN and the following parameters: a query coverage of $\geq 70 \%$ and a minimum nucleotide length of $100 \mathrm{bp}$. 


\section{Genome properties}

The genome of $A$. vaginalis strain PH9 is 2,048,125 bp long ( 1 chromosome, but no plasmid) with a $29.6 \% \mathrm{G}+\mathrm{C}$ content of (Figure 5 and Table 3 ). Of the 2,133 predicted genes, 2,095 were protein-coding genes, and 38 were RNAs. Three rRNA genes (one 16S rRNA, one 23S rRNA and one 5S rRNA) and 35 predicted tRNA genes were identified in the genome.
A total of 1,546 genes (72.48\%) were assigned a putative function. Eighty-one genes were identified as ORFans (3.8\%). The remaining genes were annotated as hypothetical proteins. The properties and the statistics of the genome are summarized in Table 3 . The distribution of genes into COGs functional categories is presented in Table 4.

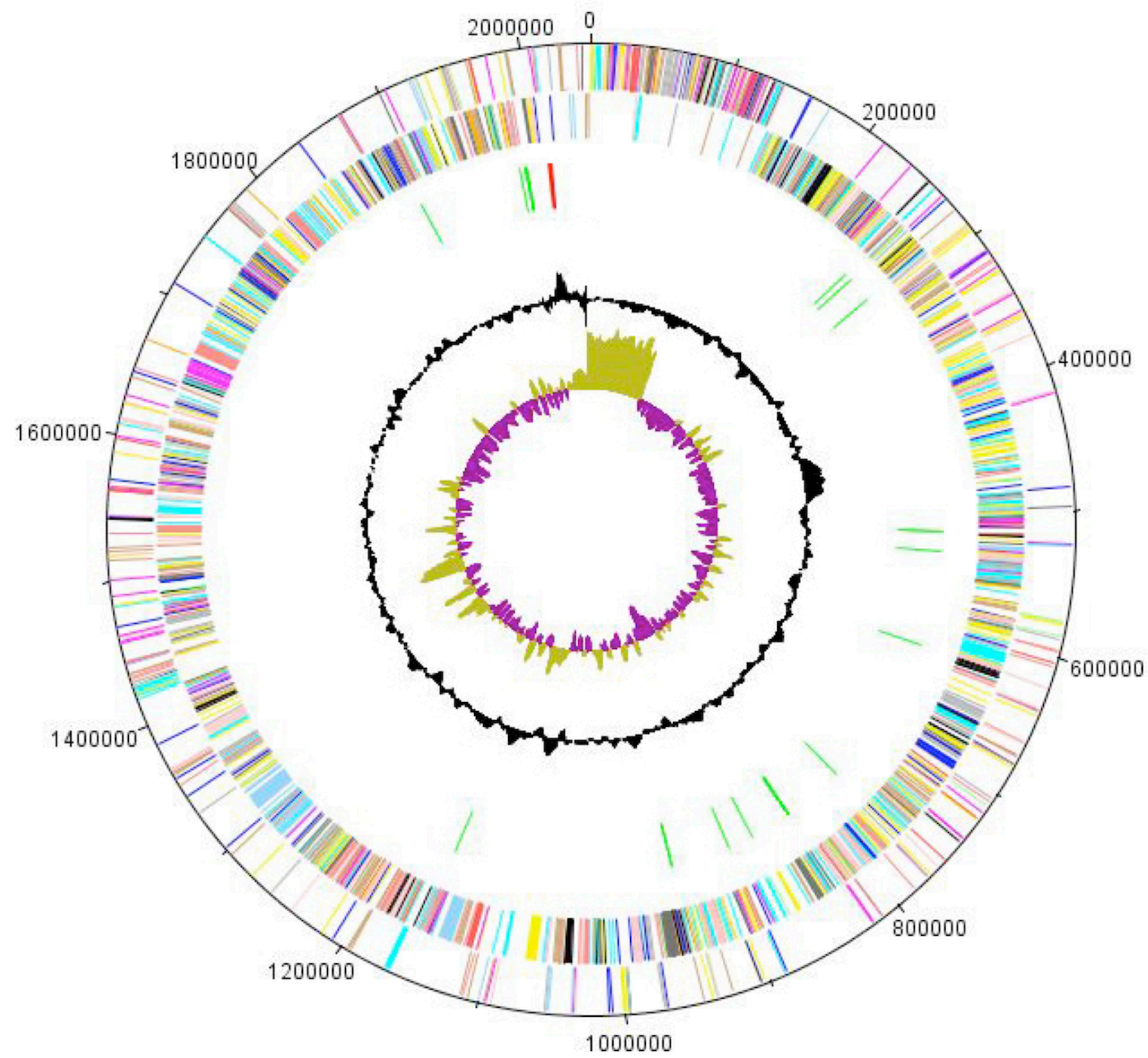

Figure 5. Graphical circular map of the chromosome. From outside to the center: Genes on the forward strand (colored by COG categories), genes on the reverse strand (colored by COG categories), RNA genes (tRNAs green, rRNAs red), GC content, and GC skew. 
Table 3. Nucleotide content and gene count levels of the genome

\begin{tabular}{lrr}
\hline Attribute & Value & \% of total \\
\hline Genome size (bp) & $2,048,125$ & \\
DNA coding region (bp) & $1,825,389$ & 89.12 \\
DNA G+C content (bp) & 593,956 & 29.60 \\
Total genes & 2,133 & 100 \\
RNA genes & 38 & 1.78 \\
Protein-coding genes & 2,095 & 98.21 \\
Genes with function prediction & 1,556 & 72.94 \\
Genes assigned to COGs & 1,546 & 72.48 \\
Genes with peptide signals & 83 & 3.89 \\
Genes with transmembrane helices & 476 & 22.31 \\
\hline
\end{tabular}

The total is based on either the size of the genome in base pairs or the total number of protein coding genes in the annotated genome.

Table 4. Number of genes associated with the 25 general COG functional categories

\begin{tabular}{crrl}
\hline Code & Value & \%age $^{\mathbf{a}}$ & Description \\
\hline J & 144 & 6.87 & Translation \\
A & 0 & 0 & RNA processing and modification \\
K & 138 & 6.59 & Transcription \\
L & 146 & 6.97 & Replication, recombination and repair \\
B & 1 & 0.05 & Chromatin structure and dynamics \\
D & 19 & 0.91 & Cell cycle control, mitosis and meiosis \\
Y & 0 & 0 & Nuclear structure \\
V & 74 & 3.53 & Defense mechanisms \\
T & 47 & 2.24 & Signal transduction mechanisms \\
M & 66 & 3.15 & Cell wall/membrane biogenesis \\
N & 5 & 0.24 & Cell motility \\
Z & 0 & 0 & Cytoskeleton \\
W & 0 & 0 & Extracellular structures \\
U & 26 & 1.24 & Intracellular trafficking and secretion \\
O & 63 & 3.01 & Posttranslational modification, protein turnover, chaperones \\
C & 90 & 4.30 & Energy production and conversion \\
G & 123 & 5.87 & Carbohydrate transport and metabolism \\
E & 136 & 6.49 & Amino acid transport and metabolism \\
F & 60 & 2.86 & Nucleotide transport and metabolism \\
H & 59 & 2.82 & Coenzyme transport and metabolism \\
I & 33 & 1.58 & Lipid transport and metabolism \\
P & 96 & 4.58 & Inorganic ion transport and metabolism \\
Q & 24 & 1.15 & Secondary metabolites biosynthesis, transport and catabolism \\
R & 206 & 9.83 & General function prediction only \\
S & 123 & 5.87 & Function unknown \\
- & 542 & 25.41 & Not in COGs \\
\hline & & & \\
\hline
\end{tabular}

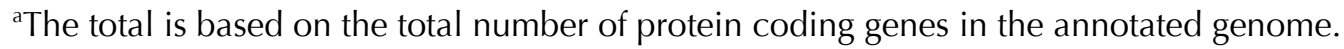




\section{Comparison with the genomes from other Anaerococcus species}

To date, two genomes from Anaerococcus species have been published. Here, we compared the genome sequence of $A$. vaginalis strain PH9 with those of $A$. prevotii strain $\mathrm{PC} 1^{\mathrm{T}}$ [24] and $A$. senegalensis strain JC48 ${ }^{\mathrm{T}}$ [25].

The draft genome sequence of $A$. vaginalis has a similar size to that of $A$. prevotii $(2.04$ vs $1.99 \mathrm{Mb}$, respectively), but a slightly larger than $A$. senegalensis $(1.79 \mathrm{Mb})$. The $\mathrm{G}+\mathrm{C}$ content of $A$. vaginalis is comparable to A. senegalensis (29.60 vs $28.56 \%$, respectively) and smaller than that of $A$. prevotii (35.64\%). The gene content of $A$. vaginalis is larger than those of $A$. prevotii and $A$. senegalensis $(2,133,1,916$ and 1,774 , respectively). The ratio of genes per $\mathrm{Mb}$ of $A$. vaginalis is larger to those of $A$. senegalensis and $A$. prevotii $(1,045,991$ and 962, respectively). Moreover, the distribution of genes into COG categories (Table 4) was highly similar in the three genomes.

\section{Reference List}

1. Ezaki T, Kawamura Y, Li N, Li ZY, Zhao L, Shu S. Proposal of the genera Anaerococcus gen. nov., Peptoniphilus gen. nov. and Gallicola gen. nov. for members of the genus Peptostreptococcus. Int J Syst Evol Microbiol 2001; 51:1521-1528. PubMed

2. Song Y, Liu C, Finegold SM. Peptoniphilus gorbachii sp. nov., Peptoniphilus olsenii sp. nov., and Anaerococcus murdochii sp. nov. isolated from clinical specimens of human origin. J Clin Microbiol 2007; 45:1746-1752. PubMed http://dx.doi.org/10.1128//CM.00213-07

3. Jain S, Bui V, Spencer C, Yee L. Septic arthritis in a native joint due to Anaerococcus prevotii. J Clin Pathol 2008; 61:775-776. PubMed http://dx.doi.org/10.1136/jcp.2007.053421

4. La Scola B, Fournier PE, Raoult D. Burden of emerging anaerobes in the MALDI-TOF and $16 \mathrm{~S}$ rRNA gene sequencing era. Anaerobe 2011; 17:106-112. PubMed http://dx.doi.org/10.1016/j.anaerobe.2011.05.010

5. Pépin J, Deslandes S, Giroux G, Sobela F, Khonde N, Diakite S, Demeule D, Labbé AC, Carrier N, Frost $\mathrm{E}$. The complex vaginal flora of west african women with bacterial vaginosis. PLOS ONE 2011; 6:e25082. PubMed http://dx.doi.org/10.1371/journal.pone.0025082
A. vaginalis shared a mean $84.8 \%$ (range 71.10 $100 \%$ ) and $88.38 \%$ (range $70.3-100 \%$ ) sequence similarity with $A$. prevotii and $A$. senegalensis respectively at the genome level.

\section{Conclusion}

We describe the phenotypic, phylogenetic and genomic characteristics of Anaerococcus vaginalis strain PH9. This bacterial strain has been found in Marseille, France.

\section{Nucleotide sequence accession numbers}

The $A$. vaginalis strain PH9 whole-genome shotgun (WGS) project and 16SrRNA gene sequence have been deposited in GenBank under accession numbers CAGU00000000 and JN837489, respectively.

6. Grice EA, Kong HH, Conlan S, Deming CB, Davis J, Young AC, NISC Comparative Sequencing Program, Bouffard GG, Blakesley RW, Murray PR, et al. Topographical and temporal diversity of the human skin microbiome. Science 2009;

324:1190-1192. PubMed http://dx.doi.org/10.1126/science. 1171700

7. Li N, Hashimoto Y, Adnan S, Miura H, Yamamoto $\mathrm{H}$, Ezaki T. Three new species of the genus Peptostreptococcus isolated from humans: Peptostreptococcus vaginalis sp. nov.,Peptostreptococcus lacrimalis sp. nov., and Peptostreptococcus lactolyticus sp. nov. Int J Syst Bacteriol 1992; 42:602-605. PubMed http://dx.doi.org/10.1099/00207713-42-4-602

8. Woese CR, Kandler O, Wheelis ML. Towards a natural system of organisms: proposal for the domains Archae, Bacteria, and Eukarya. Proc Natl Acad Sci USA 1990; 87:4576-4579. PubMed http://dx.doi.org/10.1073/pnas.87.12.4576

9. Gibbons NE, Murray RGE. Proposals concerning the higher Taxa of Bacteria. Int / Syst Bacteriol 1978; 28:1-6. http://dx.doi.org/10.1099/00207713-28-1-1

10. Garrity GM, Holt JG. The Road Map to the Manual. In: Garrity GM, Boone DR, Castenholz RW (eds), Bergey's Manual of Systematic Bacteriology, 
Second Edition, Volume 1, Springer, New York, 2001, p. 119-169.

11. Murray RGE. The Higher Taxa, or, a Place for Everything...? In: Holt JG (ed), Bergey's Manual of Systematic Bacteriology, First Edition, Volume 1, The Williams and Wilkins Co., Baltimore, 1984, p. 31-34.

12. Euzéby J. List of new names and new combinations previously effectively, but not validly, published. List no. 132. Int I Syst Evol Microbiol 2010; 60:469-472.

http://dx.doi.org/10.1099/ijs.0.022855-0

13. Rainey FA. Class II. Clostridia class nov. In: De Vos P, Garrity G, Jones D, Krieg NR, Ludwig W, Rainey FA, Schleifer KH, Whitman WB (eds), Bergey's Manual of Systematic Bacteriology, Second Edition, Volume 3, Springer-Verlag, New York, 2009, p. 736.

14. Skerman VBD, Sneath PHA. Approved list of bacterial names. Int J Syst Bact 1980; 30:225-420. http://dx.doi.org/10.1099/00207713-30-1-225

15. Prevot AR. Dictionnaire des bactéries pathogens. In: Hauduroy P, Ehringer G, Guillot G, Magrou J, Prevot AR, Rosset, Urbain A (eds). Paris, Masson, 1953, p.1-692.

16. Garrity GM, Holt J. Taxonomic outline of the Archaea and Bacteria. In: Garrity GM, Boone DR, Castenholz RW (eds), Bergey's Manual of Systematic Bacteriology. Springer-Verlag, New York, 2001, p.155-166.

17. Ashburner M, Ball CA, Blake JA, Botstein D, Butler H, Cherry JM, Davis AP, Dolinski K, Dwight SS, Eppig JT. Gene ontology: tool for the unification of biology. The Gene Ontology Consortium. Nat Genet 2000; 25:25-29. PubMed http://dx.doi.org/10.1038/75556
18. Stackebrandt E, Ebers J. Taxonomic parameters revisited: tarnished gold standards. Microbiol Today 2006; 33:152-155.

19. Seng $P$, Drancourt $M$, Gouriet F, La SB, Fournier $\mathrm{PE}$, Rolain JM. Didier Raoult. Ongoing revolution in bacteriology: routine identification of bacteria by matrix-assisted laser desorption ionization time-of-flight mass spectrometry. Clin Infect Dis 2009; 49:543-551. PubMed http://dx.doi.org/10.1086/600885

20. Prodigal. http://prodigal.ornl.gov/

21. Benson DA, Karsch-Mizrachi I, Clark K, Lipman DJ, Ostell J, Sayers EW. Gen Bank. Nucleic Acids Res 2012; 40:D48-D53. PubMed http://dx.doi.org/10.1093/nar/gkr1202

22. Lowe TM, Eddy SR. t-RNAscan-SE: a program for imroved detection of transfer RNA gene in genomic sequence. Nucleic Acids Res 1997; 25:955-964. PubMed

23. Lagesen K, Hallin P, Rodland EA, Staerfeldt HH, Rognes T, Ussery DW. RNAmmer: consistent and rapid annotation of ribosomal RNA genes. Nucleic Acids Res 2007; 35:3100-3108. PubMed http://dx.doi.org/10.1093/nar/gkm160

24. Labutti K, Pukall R, Steenblock K, Glavina Del RT, Tice H, Copeland A, Cheng JF, Lucas S, Chen $\mathrm{F}$, Nolan M. Complete genome sequence of Anaerococcus prevotii type strain (PC1). Stand Genomic Sci 2009; 1:159-165. PubMed http://dx.doi.org/10.4056/sigs.24194

25. Lagier JC, Karkouri EK, Nguyen TT, Armougom F, Raoult D, Fournier PE. Non-contiguous finished genome sequence and description of Anaerococcus senegalensis sp. nov. Stand Genomic Sci 2012; 6:116-125. PubMed http://dx.doi.org/10.4056/sigs.2415480 